\title{
Dupilumab: an evidence-based review of its potential in the treatment of atopic dermatitis
}

This article was published in the following Dove Press journal:

Core Evidence

\author{
Panteha Eshtiaghi' \\ Melinda J Gooderham ${ }^{2-4}$ \\ 'Faculty of Medicine, University \\ of British Columbia, Vancouver, \\ BC, Canada; ${ }^{2} \mathrm{SKiN}$ Centre for \\ Dermatology, Peterborough, ON, \\ Canada; ${ }^{3}$ Probity Medical Research, \\ Waterloo, ON, Canada; ${ }^{4} Q u e e n ' s$ \\ University, Kingston, ON, Canada
}

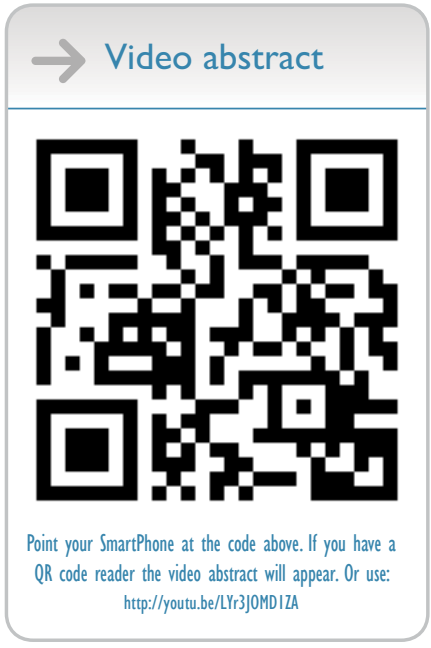

Correspondence: Melinda J Gooderham SKiN Centre for Dermatology, 775 Monaghan Road, Peterborough, ON K9] $5 \mathrm{~K} 2$, Canada

Fax +I 7057753376

Emailmgooderham@

centrefordermatology.com
Introduction: Atopic dermatitis (AD) is a recurrent, pruritic inflammatory skin disease with complex immunopathogenesis characterized by a dominant $\mathrm{T}_{\mathrm{H}} 2$ response. Dupilumab is an interleukin (IL)-4 receptor alpha antagonist that subsequently blocks IL-4 and IL-13 signaling. It has recently been approved for the treatment of adult patients with moderate-to-severe AD whose current treatment options are limited.

Aim: This article reviews the evidence of clinical efficacy, safety, and patient-reported outcome (PRO) measures from Phase I-III trials of dupilumab in adult patients with moderateto-severe AD.

Evidence review: Results from clinical trials of dupilumab in adults with moderate-to-severe AD have shown that weekly or biweekly dupilumab injections significantly improve clinical and PROs. Transcriptome and serum analyses also found that dupilumab significantly modulates the $\mathrm{AD}$ molecular signature and other $\mathrm{T}_{\mathrm{H}} 2$-associated biomarkers, compared with placebo. Additionally, concomitant use of dupilumab with topical corticosteroids (TCS) results in a greater improvement in signs and symptoms of AD than with dupilumab use alone. Throughout the trials, common adverse events were headaches, conjunctivitis, and injection site reactions. These were consistently mild-moderate and occurred with similar frequency between the treatment and placebo groups.

Place in therapy: In adult patients with moderate-to-severe refractory $\mathrm{AD}$, monotherapy or concomitant use of dupilumab with TCS holds great promise to significantly improve clinical outcomes and quality of life of the patient. Ongoing studies of dupilumab will help determine the clinical efficacy and safety profile of its long-term use. Finally, further economic evidence is warranted to compare the long-term costs and benefits of dupilumab with other currently available treatments for moderate-to-severe AD.

Keywords: moderate-to-severe atopic dermatitis, biologics, systemic therapy, interleukin-4, interleukin-13, dupilumab

\section{Core evidence clinical impact summary for dupilumab}

\begin{tabular}{|lll|}
\hline Outcome measure & Evidence & Implications \\
\hline $\begin{array}{l}\text { Disease-oriented evidence } \\
\text { Percent change in Eczema }\end{array}$ & Clinical trials & $\begin{array}{l}\text { Consistently in all trials, dupilumab treatment } \\
\text { alone and with concomitant topical } \\
\text { Area and Severity Index score }\end{array}$ \\
& & $\begin{array}{l}\text { corticosteroids use resulted in significantly more } \\
\text { participants achieving }>50 \% \text { reduction in the } \\
\end{array}$ \\
& Eczema Area and Severity Index score \\
\hline
\end{tabular}

(Continued) 


\begin{tabular}{|c|c|c|}
\hline Outcome measure & Evidence & Implications \\
\hline Disease biomarkers & Clinical trials & $\begin{array}{l}\text { Dupilumab monotherapy resulted in reduced } \\
\text { levels of thymus and activation-regulated } \\
\text { chemokine and total lgE levels in the serum. } \\
\text { Posttreatment lesional skin showed a } \\
\text { nonlesional molecular phenotype }\end{array}$ \\
\hline \multicolumn{3}{|c|}{ Patient-oriented evidence } \\
\hline Adverse events & Clinical trials & $\begin{array}{l}\text { Dupilumab therapy was well tolerated by study } \\
\text { participants. The most frequent treatment- } \\
\text { emergent adverse events were headache, } \\
\text { conjunctivitis, and injection site reaction. These } \\
\text { were transient in nature }\end{array}$ \\
\hline Pruritus and HRQoL & Clinical trials & $\begin{array}{l}\text { Dupilumab monotherapy or its concomitant } \\
\text { use with topical corticosteroids resulted } \\
\text { in significant improvement in pruritus and } \\
\text { health-related quality of life scores from } \\
\text { baseline, compared with placebo or topical } \\
\text { corticosteroids use alone. Improvement in } \\
\text { pruritus and health-related quality of life was } \\
\text { observed as early as week } 2 \text { of therapy }\end{array}$ \\
\hline \multicolumn{3}{|l|}{ Economic evidence } \\
\hline Cost-effectiveness & $\begin{array}{l}\text { Health economic } \\
\text { models }\end{array}$ & $\begin{array}{l}\text { At its current list price, dupilumab is cost- } \\
\text { effective in the treatment of adult moderate-to- } \\
\text { severe atopic dermatitis }\end{array}$ \\
\hline
\end{tabular}

\section{Introduction}

Atopic dermatitis (AD) is a chronic inflammatory skin disease characterized by pruritic, erythematous, and scaling lesions. It has a substantial effect on the quality of life for patients and caregivers, with a significant economic impact. The Global Burden of Disease 2010 Study ranked AD as the most burdensome skin condition worldwide. ${ }^{1}$ Patients with AD may be at increased risk of skin infections with systemic consequences and increased hospitalizations. ${ }^{1}$ In 2015, the annual costs of $\mathrm{AD}$ in the USA were projected to be at least $\$ 5.3$ billion, which takes into consideration direct medical costs and indirect costs of lost productivity. ${ }^{2}$

Etiology of $\mathrm{AD}$ is multifactorial, with an interplay between genetics, skin barrier function, immune dysregulation, and skin microflora. ${ }^{3}$ It is still not known whether skin inflammation arises initially from the epidermal barrier structural defects or from immune dysregulation., ${ }^{3,4}$

Recent studies have provided further insight into the immunological factors behind the pathogenesis of AD. The current understanding focuses on $A D$ as primarily a $T_{H} 2$ dominated disease, with additional roles for $\mathrm{T}_{\mathrm{H}} 22, \mathrm{~T}_{\mathrm{H}} 17$, and $\mathrm{T}_{\mathrm{H}} 1$ cytokines in certain disease subtypes. ${ }^{4}$ Cellular and molecular analyses of acute-to-chronic lesions have demonstrated a progressive increase in $\mathrm{T}_{\mathrm{H}} 2$ and $\mathrm{T}_{\mathrm{H}} 22$ gene expressions and cytokines, with simultaneous activation of $\mathrm{T}_{\mathrm{H}}$ 1-associated inflammatory response. ${ }^{5}$ Infiltrates of group 2 innate lymphoid cells in AD lesions have also been shown to promote $\mathrm{T}_{\mathrm{H}} 2$ responses by producing $\mathrm{T}_{\mathrm{H}} 2$-associated cytokines such as interleukin (IL)-4 and IL-13, ${ }^{4,5}$

IL-4 and IL-13 play an important role in the $\mathrm{T}_{\mathrm{H}}$ 2-mediated inflammatory response found in AD. IL- 4 drives the differentiation of $\mathrm{T}_{\mathrm{H}} 2$ cells and $\operatorname{IgE}$ class switching in B cells. ${ }^{6}$ Produced by $\mathrm{T}_{\mathrm{H}} 2$ cells, IL-13 is involved in B-cell maturation and differentiation and eosinophil chemotaxis and is a central mediator of allergic asthma. ${ }^{6}$ These cytokines also downregulate the expression of fundamental genes involved in skin barrier function and integrity. ${ }^{4}$ In addition, overexpression of IL-4 and IL-13 in AD prevents the upregulation of antimicrobial peptides in response to bacterial and viral stimuli in the skin. This further contributes to barrier defects and exacerbates inflammation, putting the patient at an increased risk of infection by Staphylococcus aureus. ${ }^{7}$

Current treatment is centered on nonspecific immunomodulatory topical agents such as corticosteroids and calcineurin inhibitors to prevent exacerbations and improve epidermal function. ${ }^{4}$ In patients with moderate-to-severe $\mathrm{AD}$ who have frequent flares, topical therapy is not sufficient to adequately control disease. In these patients, second-line options are restricted to immunosuppressants and ultraviolet light phototherapy. Phototherapy can be time-consuming and is not always easily accessible, and oral glucocorticoids, cyclosporine, and other immunosuppressants have long-term side effects and toxicities that limit their use. ${ }^{4}$ 
The immunopathogenesis of AD outlined provides more specific biological targets for therapy that could improve efficacy and minimize adverse effects. This article reviews a recently developed targeted therapy for $\mathrm{AD}$, including its mechanism of action, pharmacokinetics, as well as the evidence on its efficacy and safety.

\section{Dupilumab}

In March 2017, the US Food and Drug Association (FDA) approved dupilumab for the treatment of adults with moderate-to-severe $\mathrm{AD}$ whose disease is inadequately controlled by topical therapies. Dupilumab is a fully human monoclonal IgG4 antibody that binds to the shared alpha subunit of the IL-4 receptor and thereby inhibits IL-4 and IL-13 signal transduction. ${ }^{8}$ These cytokines are primarily produced by $\mathrm{T}_{\mathrm{H}} 2$ cells and are central to the pathogenesis of $\mathrm{AD}$ and other atopic diseases. ${ }^{4-6}$ In fact, dupilumab first showed its therapeutic effect in patients with moderate-to-severe asthma. ${ }^{9}$

Dupilumab's mechanism of action is best understood through serum and transcriptome analysis from clinical trials. Dupilumab has been observed to significantly reduce serum levels of thymus and activation-regulated chemokine (TARC or CCL17). ${ }^{8}$ TARC, a key regulator of $\mathrm{T}_{\mathrm{H}} 2$-mediated immunity, is considered to be a specific and objective biomarker of AD disease activity. ${ }^{10}$ In patients treated with dupilumab, tissue genomic analysis showed significant downregulation of markers associated with T-cell activation and eosinophils, as well as epidermal hyperplasia-related genes. Upregulation of barrier-related function genes was also demonstrated. ${ }^{11}$

\section{Drug formulation and dosing}

Dupilumab is administered subcutaneously. The injections can be delivered by a health care professional or caregiver or may be self-administered. ${ }^{12}$ Throughout its trials, an initial $600 \mathrm{mg}$ loading dose followed by $300 \mathrm{mg}$ injections every week or every other week was shown to be the most efficacious. ${ }^{12,13}$ Similar safety and efficacy were demonstrated in both dosing regimens within the 16-week treatment period of Phase III trials. ${ }^{12}$ Significant improvements were also seen in other patient-reported outcome (PRO) measures such as quality of life, sleep, anxiety, and depression. The trials lacked statistical power to differentiate between the two dosing regimens. ${ }^{12}$

Currently, there are limited data to comment on dose adjustments based on patient-specific factors such as age, sex, and race. It also remains to be investigated whether increasing the dose or frequency of the regimen in unresponsive patients has any additional benefits or safety concerns.

\section{Pharmacokinetics} Absorption

The bioavailability of subcutaneous dupilumab injections was determined to be $64 \% .{ }^{14}$ It takes 1 week for dupilumab to reach maximum serum concentration following a loading dose of $600 \mathrm{mg} .{ }^{14}$ In Phase III trials, steady-state concentrations were achieved by week 16 in both dose regimens. ${ }^{12}$

\section{Distribution}

The total volume of distribution of dupilumab was approximated to be $4.8 \pm 1.3 \mathrm{~L} .{ }^{14}$ This means that the drug has limited distribution, typically restricted to blood or physiological fluids.

\section{Elimination}

Following the last steady-state dose of weekly and biweekly injections, it takes a median time of 13 and 10 weeks, respectively, for dupilumab to decrease to a nondetectable concentration $(<78 \mathrm{ng} / \mathrm{mL}) .{ }^{14}$ Dupilumab is an IgG4 antibody and is thus hypothesized to be catabolized to peptides and amino acids. However, exact mechanisms of its metabolism remain unexplored. ${ }^{14}$

\section{Clinically significant drug interactions}

Formation of CYP450 enzymes is suppressed by increased levels of inflammatory cytokines. ${ }^{15}$ Dupilumab inhibits IL-4 and IL-13 receptor signaling, which could normalize levels of these enzymes. Thus, dose adjustment and drug effect monitoring of concomitant CYP450 substrates are recommended upon initiation or discontinuation of dupilumab. ${ }^{14}$

\section{Clinical efficacy}

Throughout Phase I-III trials, dupilumab has shown promising efficacy for the treatment of moderate-to-severe $\mathrm{AD}$, as assessed by specified endpoints including the Eczema Area and Severity Index (EASI), Investigator's Global Assessment (IGA; 0-4 scale) score, PROs of pruritus, and health-related quality of life (HRQoL) measures. ${ }^{8,12,13,16}$ These investigated domains represent the core outcome sets that enable pooling of data for appropriate interpretation. ${ }^{17}$ As well, the EASI is a reliable and valid outcome measurement instrument to evaluate clinical signs of $\mathrm{AD} .{ }^{17}$

In these trials, patients were assessed to have moderateto-severe $\mathrm{AD}$ if their condition had been present for at least 3 years, with EASI score $\geq 16$ and IGA scores 3 (moderate) and 4 (severe) and whose $\mathrm{AD}$ was inadequately controlled by topical treatment or in whom topical treatment was inadvisable. ${ }^{13}$ 


\section{Phase I-Ila studies}

The efficacy of dupilumab on moderate-to-severe AD was evaluated as a monotherapy in two 4-week trials (M4A and M4B) and one 12-week trial (M12) and in combination with topical corticosteroids (TCS) in another 4-week trial (C4). ${ }^{8}$ The efficacy profile was consistent across all four trials. In studies M4A and M4B, dupilumab resulted in dosedependent improvements in all efficacy endpoints, such as IGA and pruritus numerical rating scale (NRS) scores. As evaluated by M12, 85\% of patients treated with dupilumab experienced a $50 \%$ reduction in the EASI score (EASI-50) at 12 weeks compared to $35 \%$ in the placebo arm $(P<0.001)$ with the mean reduction of EASI from baseline significantly greater in the treatment group ( 74 vs $23.3 \% ; P<0.001$ ). Improvements in EASI scores were correlated with previously mentioned genomic changes. The percentage change in pruritus NRS score was also significantly reduced in patients treated with dupilumab vs placebo (55.7 vs $15.1 \% ; P<0.05)$. In combination with TCS, all 21 subjects on dupilumab reached EASI-50 by the end of the 4-week trial compared to only $5 / 10$ on TCS plus placebo $(P<0.05)$. The investigators also observed a $50 \%$ reduction in TCS use in subjects treated with combination therapy compared with those subjects on TCS and placebo $(P=0.16) .{ }^{8}$ If this noteworthy observation is maintained in the long term, dupilumab may indirectly reduce the undesirable side effect profile of long-term glucocorticoids when used in combination therapy.

\section{Phase llb study}

A subsequent double-blind placebo controlled trial of 380 patients with moderate-to-severe AD was conducted for 16 weeks. ${ }^{13}$ Safety and efficacy of multiple dose regimens of dupilumab monotherapy were assessed compared with placebo with the primary efficacy endpoint being percent change in EASI score from baseline. Subjects showed significant improvements in several endpoints in a dose-dependent manner, with $300 \mathrm{mg}$ weekly and biweekly injections producing the greatest improvement. After 16 weeks of treatment with $300 \mathrm{mg}$ dupilumab injections once a week, there was a marked least-square (LS) mean reduction of $73.7 \%$ in EASI score from baseline compared with the LS mean reduction of $18.1 \%$ observed in the placebo group $(P<0.0001)$. A total of $82.5 \%$ of subjects achieved EASI-50 and $60.3 \%$ of subjects achieved EASI-75. In contrast, only 29.5 and $11.5 \%$ of subjects in the placebo arm achieved those respective endpoints $(P<0.0001)$. These findings are consistent with those of M12. ${ }^{13}$
Furthermore, dupilumab improved PROs of pruritus and HRQoL. ${ }^{13}$ Compared to placebo, a greater proportion of subjects treated with $300 \mathrm{mg}$ dupilumab weekly and biweekly showed improvement in pruritus NRS score of $\geq 3$ at 16 weeks (54 and 41\%, respectively, vs $8 \% ; P<0.0001$ for both doses). During the 16-week treatment period, dupilumab also resulted in significant improvements $(P<0.0001)$ in HRQoL at all doses except for $100 \mathrm{mg}$ every 4 weeks, as measured by the Dermatology Quality of Life Index (DLQI). ${ }^{18}$ These findings suggest that the objective improvements observed in dupilumab-treated patients are associated with subjective improvements.

\section{Phase III studies}

Positive results of earlier studies were confirmed in two identical Phase III trials lasting 16 weeks (SOLO 1 and SOLO 2). A total of 1379 adult patients with moderate-to-severe AD were randomized to receive weekly or biweekly injections of $300 \mathrm{mg}$ dupilumab after a $600 \mathrm{mg}$ loading dose or placebo. ${ }^{12}$ At week 16, 37 and $38 \%$ of subjects in SOLO 1 achieved an IGA score of 0 or 1 and $\geq 2$-point reduction from baseline with weekly and biweekly dupilumab injections, respectively, vs $10 \%$ observed with placebo. This primary endpoint was also reached by $36 \%$ of patients in SOLO 2 with both doses compared to $8 \%$ in the placebo arm $(P<0.001$ for all comparisons). Also at 16 weeks, significantly more subjects achieved EASI-75 than those receiving placebo (SOLO 1: placebo $15 \%$, $300 \mathrm{mg}$ weekly $52 \%, 300 \mathrm{mg}$ biweekly 51\%; SOLO 2: placebo 12\%, $300 \mathrm{mg}$ weekly 48\%, $300 \mathrm{mg}$ biweekly 44\%; $P<0.001$ for all comparisons). Additionally, analysis of PROs shows a significant improvement from baseline in HRQoL scores and in pruritus as early as week 2 with dupilumab treatment compared with those on placebo $(P<0.001$ for both PROs $) .{ }^{12}$

More recently, a third Phase III trial, CHRONOS, compared the concomitant use of dupilumab and TCS therapy with placebo and TCS treatment over 52 weeks. ${ }^{16}$ The coprimary endpoints were assessed by IGA $0 / 1$ response and EASI-75 at week 16. Compared to the SOLO trials, 10-20\% more subjects achieved a $75 \%$ reduction in the EASI score at week 16 given the concomitant use of TCS in this trial. Notably, the trial showed maintenance of positive responses for 1 year. By week 52, 64\% of participants treated with $300 \mathrm{mg}$ weekly dupilumab and TCS achieved EASI-75 and $65 \%$ achieved the same co-primary endpoint on the biweekly regimen, both compared to $22 \%$ in the placebo control group $(P<0.0001)$. Significantly less proportion of participants on 
both dupilumab regimens (13 and 14\%, weekly and biweekly, respectively) suffered from AD flares through week 52 compared to the control group $(41 \% ; P<0.0001)$. Consistent with findings in C4, $300 \mathrm{mg}$ weekly dupilumab significantly reduced the proportion of days TCS and systemic rescue medications were used by participants over 16 weeks compared to placebo $(28.9$ vs $18.5 \% ; P<0.0001)$ and 52 weeks (33.7 vs $23.7 \%$; $P<0.0001$ ). With concomitant dupilumab and TCS treatment, significant improvement in pruritus was also reported starting at week 2 vs the placebo and TCS group. In participants with baseline peak pruritus NRS score $\geq 4$, the proportion who experienced an improvement of $\geq 4$ points from baseline at week 16 was significantly greater with weekly dupilumab injections (51\%) and biweekly injections $(59 \%)$ compared to control $(20 \% ; P<0.0001$ for both comparisons). This improvement was maintained at week 52. These findings were also associated with improvement in HRQoL over 52 weeks, as LS mean reduction from baseline in DLQI score was significant with both dupilumab dosing regimens $(P<0.0001){ }^{16}$

\section{Side effects}

Safety outcomes in clinical trials of dupilumab were evaluated based on incidence, type and severity of adverse events, laboratory investigations, vital signs, and electrocardiography. ${ }^{8,12,13,16}$

In early-phase trials, adverse events were described as mild to moderate and were overall similar in frequency in the placebo and treatment groups. These appeared to be transient in nature. ${ }^{8}$ Common adverse events more frequently observed in subjects receiving dupilumab were nasopharyngitis, headache, and injection site reactions. ${ }^{8}$ Although less likely to be immunogenic than chimeric mAbs, dupilumab is a human $\mathrm{mAb}$ and can still induce an antibody response. ${ }^{19}$ In a study of dupilumab in patients with persistent asthma, injectionsite reactions were more commonly reported in the treatment arm. ${ }^{9}$ In response to the same study, it has been noted that visible local reactions may inadvertently have an unmasking effect and potentially lead to observer bias. ${ }^{20}$

Serious adverse events were more commonly noted in the placebo groups, including skin infections and exacerbation of AD. ${ }^{8}$ Combined data on skin infections showed 0.05 infections per patient in the treatment group compared with 0.20 infections per patient in the placebo group. ${ }^{8}$ Patients with $\mathrm{AD}$ may be at an increased risk of bacterial, viral, and fungal skin infections. ${ }^{1}$ This suggests that if left untreated, lack of skin barrier function in AD may make patients susceptible to increased incidence of skin infections.
Similar safety results were observed in the Phase IIb study, with no dose-limiting toxic effects. ${ }^{13}$ Common side effects in the dupilumab group included nasopharyngitis, headache, and upper respiratory tract infections. In addition, participants treated with dupilumab experienced higher rates of conjunctivitis compared to placebo (7 vs 3\%). Serious adverse events were more frequently reported in the placebo group (7 vs 4\%), although the incidence of $\mathrm{AD}$ exacerbation was the same $(2 \%)$ in both the placebo arm and the combined dupilumab dosing arms. ${ }^{13}$

In two Phase III trials of dupilumab (SOLO 1 and SOLO 2), overall incidence of adverse events was comparable in the treatment and placebo groups. ${ }^{12}$ Unlike earlier published trials, higher incidence rates of nasopharyngitis were balanced across the groups. Injection site reactions were more commonly reported in the treatment groups (SOLO 1: placebo $6 \%$, weekly injections $19 \%$, biweekly injections $8 \%$; SOLO 2: placebo $6 \%$, weekly injections $13 \%$, biweekly injections $14 \%$ ). These adverse events were generally mild to moderate. In both SOLO 1 and 2, higher rates of conjunctivitis were observed in the dupilumab treatment groups (SOLO 1: $3 \%$ in weekly injections and $5 \%$ in biweekly injections vs $1 \%$ in the placebo group; SOLO 2: $4 \%$ in both weekly and biweekly injection groups vs $<1 \%$ in the placebo group). ${ }^{12}$ Interestingly, there is no report of increased incidence of conjunctivitis when dupilumab was tested in patients with asthma and nasal polyposis. ${ }^{12}$ Therefore, the pathogenesis of conjunctivitis may not be associated with dupilumab but may be a factor of AD pathogenesis itself.

Consistent with the earlier trials, skin infections and exacerbations of $\mathrm{AD}$ were more frequently seen in the placebo groups of both SOLO trials, ${ }^{12}$ suggesting improved skin barrier function in dupilumab-treated individuals. However, due to a low number of reports, there is no conclusive evidence that incidence of eczema herpeticum significantly differs between the treatment groups (SOLO 1: placebo 1\%, weekly injections $<1 \%$, biweekly injections $<1 \%$; SOLO 2 : placebo $<1 \%$, weekly injections $0 \%$, biweekly injections $1 \%){ }^{12}$ Eczema herpeticum, caused by the herpes simplex virus and barrier defects, is more common in patients with AD. ${ }^{21}$ Yet only $3 \%$ of those with $\mathrm{AD}$ develop eczema herpeticum, and the incidence is not higher in other conditions with barrier defects such as psoriasis, suggesting that it may be due to the host immune response rather than purely a barrier defect. ${ }^{21}$

More patients in the placebo group received rescue treatment than in treatment groups. ${ }^{12}$ Particularly, $8 \%$ of subjects in the placebo group received systemic corticosteroid rescue therapy compared with $2 \%$ of subjects receiving dupilumab weekly and $1 \%$ of subjects receiving injections biweekly in 
SOLO 1. Similarly, in SOLO 2, 13\% of subjects in the placebo group received systemic corticosteroid rescue therapy and only 3 and $1 \%$ of subjects in the other two respective dupilumab dosing regimens. ${ }^{12}$ These findings further support the fact that dupilumab may reduce the need for systemic corticosteroids and the burden of their side effects.

Analysis of safety outcomes in the CHRONOS trial revealed a similar overall proportion of patients experiencing adverse events in both the experimental and control groups: $83 \%$ with weekly dupilumab, $88 \%$ with biweekly dupilumab, and $84 \%$ with placebo. ${ }^{16}$ In line with earlier findings, increased incidence of injection site reactions (19\% weekly and $15 \%$ biweekly vs $8 \%$ placebo) and conjunctivitis (19\% weekly and $14 \%$ biweekly vs $8 \%$ placebo) were the most commonly reported adverse events in the dupilumab and TCS treatment groups. The rate of nonherpetic skin infections was again reduced in this study with weekly ( $8 \%)$ and biweekly (11\%) dupilumab treatment plus TCS compared to placebo plus TCS therapy (18\%). Comparable to the previous trials, higher rates of serious adverse events were reported in the control group and $58 \%$ of participants in this arm who discontinued the trial withdrew due to flaring of their AD. ${ }^{16}$

Of note, there were a total of three deaths in the treatment groups of SOLO 2 and CHRONOS trials. None were reported to be related to the study drug. ${ }^{12}$

The preceding trials demonstrate a favorable and consistent safety profile of dupilumab alone, and with concomitant TCS, in patients with moderate-to-severe AD. However, long-term data are required to conclusively determine its safety and tolerability.

\section{Clinical indications}

Dupilumab has been approved for use in the management of moderate-to-severe refractory adult AD. Dupilumab can be used in combination with or without topical corticosteroids. Topical calcineurin inhibitors may also be used in areas such as face, neck, intertriginous, and genital areas. ${ }^{14}$

\section{Cost-effectiveness}

Economic studies on the use of dupilumab in the treatment of moderate-to-severe adult $\mathrm{AD}$ are limited to two cost-effectiveness analyses. One recent study model determined that biweekly dupilumab injections was cost-effective compared to supportive care (standard emollients), at an annual drug price in the range of $\$ 29,000-\$ 40,000$ per quality-adjusted life-years (QALY) gained with respect to a $\$ 100,000-\$ 150,000$ per QALY threshold. Currently, the annual list price for dupilumab is $\$ 37,000$, which falls within this range. The thresholds used are acceptable cost- effectiveness decision thresholds in the USA. ${ }^{22}$ Similarly, a study model by the Institute for Clinical and Economic Review also found dupilumab to be a cost-effective treatment compared to the usual standard of care, with an annual price of \$30,516-\$43,726 per QALY gained at the same thresholds. Usual care did not include phototherapy or systemic immunomodulatory treatment modalities. ${ }^{23}$ Further studies are required to compare the cost of dupilumab with conventional therapies.

\section{Ongoing studies}

The pediatric population bears the highest prevalence of AD worldwide. ${ }^{3}$ Clinical trials are currently underway to evaluate the safety and efficacy of dupilumab in patients aged $\geq 6-<18$ years (NCT02407756, NCT02612454) and $\geq 12-<18$ years (NCT03054428) who suffer from moderateto-severe AD. Effective treatments for AD in early childhood could potentially prevent or slow the progression to other atopic diseases such as asthma and allergic rhinitis, otherwise known as the atopic march. ${ }^{24}$ Another Phase III trial is ongoing to evaluate the efficacy and safety of dual dupilumab and TCS therapy in patients with severe $\mathrm{AD}$, inadequately controlled by oral Cyclosporine A (Café study, NCT02755649). Importantly, SOLO-CONTINUE is monitoring long-term response maintenance in SOLO participants who achieved either IGA $=0$ or 1 or EASI-75 at week 16 (NCT02395133) and the delivery of dupilumab by an auto-injector device is being compared to delivery by prefilled syringe in moderate-to-severe AD subjects aged 12 years and older (NCT03050151).

\section{Future directions}

$\mathrm{AD}$ is a chronic condition and thus poor adherence to treatment is common. Future investigations should also examine the efficacy of dupilumab if the drug regimen is interrupted or withdrawn and re-introduced. Studies have yet to report withdrawal effects and whether drug discontinuation will result in rebound flaring or $\mathrm{AD}$ exacerbation and what is the recapture rate when dupilumab is re-introduced.

Additionally, population studies have shown different AD molecular signatures among various races. ${ }^{25}$ For instance, along with a $\mathrm{T}_{\mathrm{H}} 2$ phenotype, studies have shown a $\mathrm{T}_{\mathrm{H}} 17$ cytokine profile in Asian patients with AD. Therefore, therapies that also target IL-17/IL-23 may have some benefit in such patients. ${ }^{25}$ Although transcriptome analysis following dupilumab treatment demonstrated decreased expression of IL-17-modulated genes, IL-17A mRNA expression was not reduced. ${ }^{11}$ The Phase III clinical trials included participants of Asian descent so a subgroup analysis may provide 
valuable information about the treatment response in these participants.

Other Th2 cytokines have been the target of emerging biological agents for the treatment of AD. A recent metaanalysis assessed the evidence behind efficacy and safety of these biologics in patients with moderate-to-severe AD. ${ }^{26}$ Lebrikizumab and tralokinumab are monoclonal antibodies against IL-13. In a Phase II study, monthly lebrikizumab injections with concomitant TCS use for 12 weeks resulted in a superior primary outcome, EASI-50 score, compared to placebo. Biweekly tralokinumab and concomitant TCS use for 12 weeks significantly reduced absolute change in EASI from baseline compared to placebo and a greater proportion of patients treated with tralokinumab had an IGA response of 0 or $1 .{ }^{27}$ Furthermore, nemolizumab is a monoclonal antibody against the IL-31 receptor A, another target in the Th2 axis. IL-31 mediates pruritus and inflammation and has been correlated with disease severity. ${ }^{28}$ In a Phase II trial, nemolizumab significantly improved pruritus as the primary outcome. However, it was not shown to be superior to placebo with respect to EASI-75. ${ }^{26}$ Larger and longer trials are needed to adequately assess adverse events and long-term efficacy of the aforementioned agents. Dupilumab is the only biological agent to date that has shown consistent efficacious and safety outcomes.

\section{Conclusion}

$\mathrm{AD}$ is a complex disorder, and its severe forms are more difficult to treat. Many available treatment options are not selective and, as such, have undesirable side effects. Increase in the knowledge of the specific immune axes involved in $\mathrm{AD}$ pathogenesis has been essential in the development of monoclonal antibodies against key perpetrators. Dupilumab is the first such approved targeted biologic therapy. If it demonstrates sustained efficacy and safety in long-term studies, it will be a successful treatment option for adult patients with moderate-to-severe AD. Nevertheless, dupilumab clinical trials to date have been essential in further understanding the pathophysiology of this condition and have opened up new avenues for advances in AD immunotherapy.

\section{Disclosure}

MJG has been an investigator, speaker, consultant, and/or advisory board member for AbbVie, Amgen, Boehringer Ingelheim, Celgene, Dermira, Eli Lilly, Galderma, GSK, Janssen, Kyowa Kirin, Leo Pharma, Medimmune, Novartis, Pfizer, Roche, Regeneron, Sanofi Genzyme, UCB, and Valeant. PE reports no conflicts of interest in this work.

\section{References}

1. Drucker AM. At dermatitis: burden of illness, quality of life, and associated complications. Allergy Asthma Proc. 2017;38(1):3-8.

2. Drucker AM, Wang AR, Li W-Q, Sevetson E, Block JK, Qureshi AA. The burden of atopic dermatitis: summary of a report for the National Eczema Association. J Invest Dermatol. 2016;137(1):26-30.

3. Boguniewicz M, Leung DYM. Atopic dermatitis: a disease of altered skin barrier and immune dysregulation. Immunol Rev. 2011;242(1): 233-246.

4. Brunner PM, Guttman-Yassky E, Leung DYM. The immunology of atopic dermatitis and its reversibility with broad-spectrum and targeted therapies. J Allergy Clin Immunol. 2017;139(Suppl 4):S65-S76.

5. Bullens DM, Kasran A, Peng X, Lorré K, Ceuppens JL. Clinical and experimental immunology: effects of anti-IL-4 receptor monoclonal antibody on in vitro T cell cytokine levels: IL-4 production by T cells from non-atopic donors. Clin Exp Immunol. 1998;113(3):320-326.

6. Gandhi NA, Pirozzi G, Graham NMH. Commonality of the IL-4/ IL-13 pathway in atopic diseases. Expert Rev Clin Immunol. 2017; 13(5):1-13.

7. Kisich KO, Carspecken CW, Fiéve S, Boguniewicz M, Leung DYM. Defective killing of Staphylococcus aureus in atopic dermatitis is associated with reduced mobilization of human $\beta$-defensin-3. J Allergy Clin Immunol. 2008;122(1):62-68.

8. Beck LA, Thaci D, Hamilton JD, et al. Dupilumab treatment in adults with moderate-to-severe atopic dermatitis. $N$ Engl $J$ Med. 2014;371(2):130-139.

9. Wenzel S, Ford L, Pearlman D, et al. Dupilumab in persistent asthma with elevated eosinophil levels. $N$ Engl J Med. 2013;368(26): 2455-2466.

10. Kakinuma T, Nakamura K, Wakugawa M, et al. Thymus and activationregulated chemokine in atopic dermatitis: serum thymus and activationregulated chemokine level is closely related with disease activity. J Allergy Clin Immunol. 2001;107(3):535-541.

11. Hamilton JD, Suárez-Fariñas M, Dhingra N, et al. Dupilumab improves the molecular signature in skin of patients with moderateto-severe atopic dermatitis. J Allergy Clin Immunol. 2014;134(6): 1293-1300.

12. Simpson EL, Bieber T, Guttman-Yassky E, et al. Two phase 3 trials of dupilumab versus placebo in atopic dermatitis. NEngl J Med. 2016;375(24): 2335-2348.

13. Thaçi D, Simpson EL, Beck LA, et al. Efficacy and safety of dupilumab in adults with moderate-to-severe atopic dermatitis inadequately controlled by topical treatments: a randomised, placebo-controlled, dose-ranging phase 2b trial. Lancet. 2016;387(10013):40-52.

14. DUPIXENT ${ }^{\circledR}$ [package insert]. Tarrytown, NY: Regeneron Pharmaceuticals; 2017.

15. Huang S-M, Zhao H, Lee J-I, et al. Therapeutic protein-drug interactions and implications for drug development. Clin Pharmacol Ther. 2010;87(4):497-503.

16. Blauvelt A, de Bruin-Weller M, Gooderham M, et al. Long-term management of moderate-to-severe atopic dermatitis with dupilumab and concomitant topical corticosteroids (LIBERTY AD CHRONOS): a 1 -year, randomised, double-blinded, placebo-controlled, phase 3 trial. Lancet. 2017;389(10086):2287-2303.

17. Schmitt J, Apfelbacher C, Spuls PI, et al. The harmonizing outcome measures for eczema (HOME) roadmap: a methodological framework to develop core sets of outcome measurements in dermatology. J Invest Dermatol. 2015;135(1):24-30.

18. Simpson EL, Gadkari A, Worm M, et al. Dupilumab therapy provides clinically meaningful improvement in patient-reported outcomes (PROs): a phase IIb, randomized, placebo-controlled, clinical trial in adult patients with moderate to severe atopic dermatitis (AD). $J \mathrm{Am}$ Acad Dermatol. 2016;75(3):506-515.

19. Hwang WYK, Foote J. Immunogenicity of engineered antibodies. Methods. 2005;36(1):3-10.

20. Bjarnason N. [letter]. N Engl J Med. 2013;368(26):2455-2466. 
21. Ong PY, Leung DYM. Bacterial and viral infections in atopic dermatitis: a comprehensive review. Clin Rev Allergy Immunol. 2016;51(3): 329-337.

22. Kuznik A, Bégo-Le-Bagousse G, Eckert L, et al. Economic evaluation of dupilumab for the treatment of moderate-to-severe atopic dermatitis in adults. 2017;7:493-505.

23. Institute for Clinical and Economic Review. Dupilumab and Crisaborole for Atopic Dermatitis: Effectiveness and Value. Evidence Report; 2017. Available from: https://icer-review.org/wp-content/uploads/2016/10/ MWCEPAC_ATOPIC_EVIDENCE_REPORT_051217.pdf. Accessed January 6, 2018.

24. Zheng T, Yu J, Oh MH, Zhu Z. The atopic march: progression from atopic dermatitis to allergic rhinitis and asthma. Allergy Asthma Immunol Res. 2011;3(2):67-73.
25. Werfel T, Allam JP, Biedermann T, et al. Cellular and molecular immunologic mechanisms in patients with atopic dermatitis. J Allergy Clin Immunol. 2016;138(2):336-349.

26. Snast I, Reiter O, Hodak E, Friedland R, Mimouni D, Leshem YA. Are biologics efficacious in atopic dermatitis? A systematic review and meta-analysis. Am J Clin Dermatol. Epub 2017 Nov 2.

27. Wollenberg A, Howell MD, Guttman-yassky E, et al. A Phase 2b DoseRanging Efficacy and Safety Study of Tralokinumab in Adult Patients with Moderate to Severe Atopic Dermatitis (AD). Poster presented at: American Academy of Dermatology (AAD) $75^{\text {th }}$ Annual Meeting; March 3, 2017; Orlando, FL.

28. Raap U, Wichmann K, Bruder M, et al. Correlation of IL-31 serum levels with severity of atopic dermatitis. JAllergy Clin Immunol. 2008;122(2): $421-423$.

\section{Core Evidence}

\section{Publish your work in this journal}

Core Evidence is an international, peer-reviewed open-access journal evaluating the evidence underlying the potential place in therapy of drugs throughout their development lifecycle from preclinical to post launch. The focus of each review is to evaluate the case for a new drug or class in outcome terms in specific indications and patient groups.

Submit your manuscript here: https://www.dovepress.com/core-evidence-journal
Dovepress

The manuscript management system is completely online and includes a very quick and fair peer-review system, which is all easy to use. Visit http://www.dovepress.com/testimonials.php to read real quotes from published authors. 